TPLP 20 (5): 751-766, 2020. (C) The Author(s), 2020. Published by Cambridge University Press.

This is an Open Access article, distributed under the terms of the Creative Commons Attribution licence (http://creativecommons.org/licenses/by/4.0/), which permits unrestricted re-use, distribution, and reproduction in any medium, provided the original work is properly cited.

doi:10.1017/S1471068420000381

\title{
An ASP approach for reasoning in a concept-aware multipreferential lightweight $D L$
}

\author{
Laura Giordano, Daniele Theseider Dupré \\ DISIT, Università del Piemonte Orientale, Italy \\ (e-mail: laura.giordano@uniupo.it, dtd@uniupo.it)
}

submitted 10 August 2020; revised 10 August 2020; accepted 12 August 2020

\begin{abstract}
In this paper we develop a concept aware multi-preferential semantics for dealing with typicality in description logics, where preferences are associated with concepts, starting from a collection of ranked TBoxes containing defeasible concept inclusions. Preferences are combined to define a preferential interpretation in which defeasible inclusions can be evaluated. The construction of the concept-aware multipreference semantics is related to Brewka's framework for qualitative preferences. We exploit Answer Set Programming (in particular, asprin) to achieve defeasible reasoning under the multipreference approach for the lightweight description logic $\mathcal{E} \mathcal{L}_{\perp}^{+}$.
\end{abstract}

KEYWORDS: Nonmonotonic Reasoning, Description Logics, Preferences, ASP

\section{Introduction}

The need to reason about exceptions in ontologies has led to the development of many non-monotonic extensions of Description Logics (DLs), incorporating features from NMR formalisms in the literature (Straccia 1993; Baader and Hollunder 1995; Donini et al. 2002; Giordano et al. 2007; Britz et al. 2008; Bonatti et al. 2009; Casini and Straccia 2010; Motik and Rosati 2010), and notably including extensions of rule-based languages (Eiter et al. 2008; Eiter et al. 2011; Knorr et al. 2012; Gottlob et al. 2014; Giordano and Theseider Dupré 2016; Bozzato et al. 2018), as well as new constructions and semantics (Casini and Straccia 2013; Bonatti et al. 2015; Bonatti 2019). Preferential approaches (Kraus et al. 1990; Lehmann and Magidor 1992) have been extended to description logics, to deal with inheritance with exceptions in ontologies, allowing for non-strict forms of inclusions, called typicality or defeasible inclusions, with different preferential semantics (Giordano et al. 2007; Britz et al. 2008) and closure constructions (Casini and Straccia 2010; Casini et al. 2013; Giordano et al. 2013; Pensel and Turhan 2018).

In this paper, we propose a "concept-aware multipreference semantics" for reasoning about exceptions in ontologies taking into account preferences with respect to different concepts and integrating them into a preferential semantics which allows a standard interpretation of defeasible inclusions. The intuitive idea is that the relative typicality of two domain individuals usually depends on the aspects we are considering for comparison: Bob may be a more typical as sport lover than Jim, but Jim may be a more typical swimmer than Bob. This leads to consider a multipreference semantics in which there is 
a preference relation $\leq_{C}$ among individuals for each aspect (concept) $C$. In the previous case, we would have bob $\leq_{\text {SportLover }} j \mathrm{im}$ and $j i m \leq_{\text {Swimmer }}$ bob. Considering different preference relations associated with concepts, and then combining them into a global preference, provides a simple solution to the blocking inheritance problem, which affects rational closure, while still allowing to deal with specificity and irrelevance.

Our approach is strongly related with Gerard Brewka's proposal of preferred subtheories (Brewka 1989), later generalized within the framework of Basic Preference Descriptions for ranked knowledge bases (Brewka 2004). We extend to DLs the idea of having ranked or stratified knowledge bases (ranked TBoxes here) and to define preorders (preferences) on worlds (here, preferences among domain elements in a DL interpretation). Furthermore, we associate ranked TBoxes with concepts. The ranked TBox for concept $C$ describes the prototypical properties of $C$-elements. For instance, the ranked TBox for concept Horse describes the typical properties of horses, of running fast, having a long mane, being tall, having a tail and a saddle. These properties are defeasible and horses should not necessarily satisfy all of them.

The ranked TBox for $C_{h}$ determines a preference relation $\leq_{C_{h}}$ on the domain, defining the relative typicality of domain elements with respect to aspect $C_{h}$. We then combine such preferences into a global preference relation $<$ to define a concept-wise multipreference semantics, in which all conditional queries can be evaluated as usual in preferential semantics. For instance, we may want to check whether typical Italian employees have a boss, given the preference relation $\leq_{\text {Employee }}$, but no preference relation for concept Italian; or to check whether employed students are normally young or have a boss, given the preference relations $\leq_{\text {Employee }}$ and $\leq_{\text {Student }}$, resp., for employees and for students.

We introduce a notion of multipreference entailment and prove that it satisfies the KLM properties of preferential consequence relations. This notion of entailment deals properly with irrelevance and specificity, is not subject to the "blockage of property inheritance" problem, which affects rational closure (Pearl 1990), i.e., if a subclass is exceptional with respect to a superclass for a given property, it does not inherit from that superclass any other property.

To prove the feasibility of our approach, we develop a proof method for reasoning under the proposed multipreference semantics for the description logic $\mathcal{E} \mathcal{L}_{\perp}^{+}$(Kazakov et al. 2014), the fragment of OWL2 EL supported by ELK. We reformulate multipreference entailment as a problem of computing preferred answer sets and, as a natural choice, we develop an encoding of the multipreferential extension of $\mathcal{E} \mathcal{L}_{\perp}^{+}$in asprin (Brewka et al. 2015), exploiting a fragment of Krötzsch's Datalog materialization calculus (2010).

As a consequence of the soundness and completeness of this reformulation of multipreference entailment, we prove that concept-wise multipreference entailment is $\Pi_{2}^{p}$-complete for $\mathcal{E L}_{\perp}^{+}$ranked knowledge bases.

\section{Preliminaries: The description logics $\mathcal{E} \mathcal{L}_{\perp}^{+}$}

We consider the description logic $\mathcal{E} \mathcal{L}_{\perp}^{+}$(Kazakov et al. 2014) of the $\mathcal{E} \mathcal{L}$ family (Baader et al. 2005). Let $N_{C}$ be a set of concept names, $N_{R}$ a set of role names and $N_{I}$ a set of individual names. The set of $\mathcal{E L}_{\perp}^{+}$concepts can be defined as follows: $C:=A|\top| \perp \mid$ $C \sqcap C \mid \exists r . C$, where $a \in N_{I}, A \in N_{C}$ and $r \in N_{R}$. Observe that union, complement and universal restriction are not $\mathcal{E} \mathcal{L}_{\perp}^{+}$constructs. A knowledge base $(\mathrm{KB}) K$ is a pair $(\mathcal{T}, \mathcal{A})$, 
where $\mathcal{T}$ is a TBox and $\mathcal{A}$ is an ABox. The TBox $\mathcal{T}$ is a set of concept inclusions (or subsumptions) of the form $C \sqsubseteq D$, where $C, D$ are concepts, and of role inclusions of the form $r_{1} \circ \ldots \circ r_{n} \sqsubseteq r$, where $r_{1}, \ldots, r_{n}, r \in N_{R}$. The ABox $\mathcal{A}$ is a set of assertions of the form $C(a)$ and $r(a, b)$ where $C$ is a concept, $r \in N_{R}$, and $a, b \in N_{I}$.

An interpretation for $\mathcal{E} \mathcal{L}_{\perp}^{+}$is a pair $I=\left\langle\Delta,{ }^{I}\right\rangle$ where: $\Delta$ is a non-empty domain-a set whose elements are denoted by $x, y, z, \ldots$ - and $\cdot^{I}$ is an extension function that maps each concept name $C \in N_{C}$ to a set $C^{I} \subseteq \Delta$, each role name $r \in N_{R}$ to a binary relation $r^{I} \subseteq \Delta \times \Delta$, and each individual name $a \in N_{I}$ to an element $a^{I} \in \Delta$. It is extended to complex concepts as follows: $\top^{I}=\Delta, \perp^{I}=\emptyset,(C \sqcap D)^{I}=C^{I} \cap D^{I}$ and $(\exists r . C)^{I}=\left\{x \in \Delta \mid \exists y .(x, y) \in r^{I}\right.$ and $\left.y \in C^{I}\right\}$. The notions of satisfiability of a $\mathrm{KB}$ in an interpretation and of entailment are defined as usual:

Definition 1 (Satisfiability and entailment)

Given an $\mathcal{E} \mathcal{L}_{\perp}^{+}$interpretation $I=\left\langle\Delta,{ }^{I}\right\rangle$ :

- $I$ satisfies an inclusion $C \sqsubseteq D$ if $C^{I} \subseteq D^{I}$;

- I satisfies a role inclusions $r_{1} \circ \ldots \circ r_{n} \sqsubseteq r$ if $r_{1}^{I} \circ \ldots \circ r_{n}^{I} \subseteq r^{I}$;

- $I$ satisfies an assertion $C(a)$ if $a^{I} \in C^{I}$ and an assertion $r(a, b)$ if $\left(a^{I}, b^{I}\right) \in r^{I}$.

Given a $\mathrm{KB} K=(\mathcal{T}, \mathcal{A})$, an interpretation $I$ satisfies $\mathcal{T}$ (resp. $\mathcal{A}$ ) if $I$ satisfies all inclusions in $\mathcal{T}$ (resp. all assertions in $\mathcal{A}$ ); $I$ is a model of $K$ if $I$ satisfies $\mathcal{T}$ and $\mathcal{A}$.

A subsumption $F=C \sqsubseteq D$ (resp., an assertion $C(a), R(a, b)$ ), is entailed by $K$, written $K \models F$, if for all models $I=\left\langle\Delta, \cdot^{I}\right\rangle$ of $K, I$ satisfies $F$.

\section{Multiple preferences from ranked TBoxes}

To define a multipreferential semantics for $\mathcal{E} \mathcal{L}_{\perp}^{+}$we extend the language with a typicality operator $\mathbf{T}$, as done for $\mathcal{E L}^{\perp}$ (Giordano et al. 2011). In the language extended with the typicality operator, an additional concept $\mathbf{T}(C)$ is allowed (where $C$ is an $\mathcal{E L}_{\perp}^{+}$ concept), whose instances are intended to be the prototypical instances of concept $C$. Here, we assume that $\mathbf{T}(C)$ can only occur on the left hand side of concept inclusion, to allow typicality inclusions of the form $\mathbf{T}(C) \sqsubseteq D$, meaning that "typical C's are D's" or "normally C's are D's". Such inclusions are defeasible, i.e., admit exceptions, while standard inclusions are called strict, and must be satisfied by all domain elements.

Let $\mathcal{C}$ be a (finite) set of distinguished concepts $\left\{C_{1}, \ldots, C_{k}\right\}$, where $C_{1}, \ldots, C_{k}$ are possibly complex $\mathcal{E} \mathcal{L}_{\perp}^{+}$concepts. Inspired to Brewka's framework of basic preference descriptions (Brewka 2004), we introduce a ranked $T B o x \mathcal{T}_{C_{i}}$ for each concept $C_{i} \in$ $\mathcal{C}$, describing the typical properties $\mathbf{T}\left(C_{i}\right) \sqsubseteq D$ of $C_{i}$-elements. Ranks (non-negative integers) are assigned to such inclusions; the ones with higher rank are considered more important than the ones with lower rank.

A ranked $\mathcal{E} \mathcal{L}_{\perp}^{+}$knowledge base $K$ over $\mathcal{C}$ is a tuple $\left\langle\mathcal{T}_{\text {strict }}, \mathcal{T}_{C_{1}}, \ldots, \mathcal{T}_{C_{k}}, \mathcal{A}\right\rangle$, where $\mathcal{T}_{\text {strict }}$ is a set of standard concept and role inclusions, $\mathcal{A}$ is an ABox and, for each $C_{j} \in \mathcal{C}, \mathcal{T}_{C_{j}}$ is a ranked TBox of defeasible inclusions, $\left\{\left(d_{i}^{j}, r_{i}^{j}\right)\right\}$, where each $d_{i}^{j}$ is a typicality inclusion of the form $\mathbf{T}\left(C_{j}\right) \sqsubseteq D_{i}^{j}$, having rank $r_{i}^{j}$, a non-negative integer.

\section{Example 1}

Consider the ranked $\mathrm{KB} K=\left\langle\mathcal{T}_{\text {strict }}, \mathcal{T}_{\text {Horse }}, \mathcal{A}\right\rangle$ (with empty $\mathcal{A}$ ), where $\mathcal{T}_{\text {strict }}$ contains Horse $\sqsubseteq$ Mammal, Mammal $\sqsubseteq$ Animal, and $\mathcal{T}_{\text {Horse }}=\left\{\left(d_{1}, 0\right),\left(d_{2}, 0\right),\left(d_{3}, 1\right),\left(d_{4}, 2\right)\right\}$ where the defeasible inclusions $d_{1}, \ldots, d_{4}$ are as follows: 
$\left(d_{1}\right) \mathbf{T}($ Horse $) \sqsubseteq \exists$ has_equipment.Saddle

$\left(d_{2}\right) \mathbf{T}$ (Horse) $\sqsubseteq \exists$ Has_Mane.Long

$\left(d_{3}\right) \mathbf{T}($ Horse $) \sqsubseteq$ RunFast

$\left(d_{4}\right) \mathbf{T}$ (Horse) $\sqsubseteq \exists$ Has_Tail. $\top$

The ranked Tbox $\mathcal{T}_{\text {Horse }}$ can be used to define an ordering among domain elements comparing their typicality as horses. For instance, given two horses Spirit and Buddy, if Spirit has long mane, no saddle, has a tail and runs fast, it is intended to be more typical than Buddy, a horse running fast, with saddle and long mane, but without tail, as having a tail (rank 2) is a more important property for horses wrt having a saddle (rank 0).

In order to define an ordering for each $C_{i} \in \mathcal{C}$, where $x \leq_{C_{i}} y$ means that $x$ is at least as typical as $y$ wrt $C_{i}$ (in the example, Spirit $\leq_{\text {Horse }}$ Buddy and, actually, Spirit $<_{\text {Horse }}$ Buddy), among the preference strategies considered by Brewka, we adopt strategy \#, which considers the number of formulas satisfied by a domain element for each rank.

Given a ranked knowledge base $K=\left\langle\mathcal{T}_{\text {strict }}, \mathcal{T}_{C_{1}}, \ldots, \mathcal{T}_{C_{k}}, \mathcal{A}\right\rangle$, where $\mathcal{T}_{C_{j}}=\left\{\left(d_{i}^{j}, r_{i}^{j}\right)\right\}$ for all $j=1, \ldots, k$, let us consider an $\mathcal{E L}_{\perp}^{+}$interpretation $I=\left\langle\Delta,{ }^{I}\right\rangle$ satisfying all the strict inclusions in $\mathcal{T}_{\text {strict }}$ and assertions in $\mathcal{A}$. For each $j$, to define a preference ordering $\leq_{C_{j}}$ on $\Delta$, we first need to determine when a domain element $x \in \Delta$ satisfies/violates a typicality inclusion for $C_{j}$. We say that $x \in \Delta$ satisfies $\mathbf{T}\left(C_{j}\right) \sqsubseteq D$ in $I$, if $x \notin C_{j}^{I}$ or $x \in D^{I}$, while $x$ violates $\mathbf{T}\left(C_{j}\right) \sqsubseteq D$ in $I$, if $x \in C_{j}^{I}$ and $x \notin D^{I}$. Note that any element which is not an instance of $C_{j}$ trivially satisfies all conditionals $\mathbf{T}\left(C_{j}\right) \sqsubseteq D_{i}^{j}$. For a domain element $x \in \Delta$, let $\mathcal{T}_{C_{j}}^{l}(x)$ be the set of typicality inclusions in $\mathcal{T}_{C_{j}}$ with rank $l$ which are satisfied by $x: \mathcal{T}_{C_{j}}^{l}(x)=\left\{d \mid(d, l) \in \mathcal{T}_{C_{j}}\right.$ and $x$ satisfies $d$ in $\left.I\right\}$.

Definition $2\left(\leq_{C_{j}}\right)$

Given a ranked knowledge base $K$ as above and an $\mathcal{E} \mathcal{L}_{\perp}^{+}$interpretation $I=\left\langle\Delta,{ }^{I}\right\rangle$, the preference relation $\leq_{C_{j}}$ associated with $\mathcal{T}_{C_{j}}=\left\{\left(D_{i}^{j}, r_{i}^{j}\right)\right\}$ in $I$ is defined as follows:

$$
\begin{aligned}
& x_{1} \leq C_{j} x_{2} \quad \text { iff either }\left|\mathcal{T}_{C_{j}}^{l}\left(x_{1}\right)\right|=\left|\mathcal{T}_{C_{j}}^{l}\left(x_{2}\right)\right| \text { for all } l, \\
& \quad \text { or } \exists l \text { such that }\left|\mathcal{T}_{C_{j}}^{l}\left(x_{1}\right)\right|>\left|\mathcal{T}_{C_{j}}^{l}\left(x_{2}\right)\right| \text { and, } \forall h>l,\left|\mathcal{T}_{C_{j}}^{h}\left(x_{1}\right)\right|=\left|\mathcal{T}_{C_{j}}^{h}\left(x_{2}\right)\right|
\end{aligned}
$$

A strict preference relation $<_{C_{j}}$ and an equivalence relation $\sim_{C_{j}}$ can be defined as usual letting: $x_{1}<_{C_{j}} x_{2}$ iff $\left(x_{1} \leq_{C_{j}} x_{2}\right.$ and not $\left.x_{2} \leq_{C_{j}} x_{1}\right)$, and $x \sim_{C_{j}} y$ iff $\left(x \leq_{C_{j}} y\right.$ and $\left.y \leq_{C_{j}} x\right)$.

Informally, $\leq_{C_{j}}$ gives higher preference to domain individuals violating less typicality inclusions with higher rank. Definition 2 exploits Brewka's \# strategy in DL context. In particular, all $x, y \notin C_{j}^{I}, x \sim_{C_{j}} y$, i.e., all elements not belonging to $C_{j}^{I}$ are assigned the same rank, the least one, as they trivially satisfy all the typical properties of $C_{j}$ 's. As, for a ranked knowledge base, the \# strategy defines a total preorder (Brewka 2004) and, for each $\mathcal{T}_{C_{j}}$, we have applied this strategy to the materializations $C_{j} \sqsubseteq D$ of the typicality inclusions $\mathbf{T}\left(C_{j}\right) \sqsubseteq D$ in the ranked TBox $\mathcal{T}_{C_{j}}$, the relation $\leq_{C_{j}}$ is a total preorder on the domain $\Delta$. Then, the strict preference relation $<_{C_{j}}$ is a strict modular partial order, i.e., an irreflexive, transitive and modular relation (where modularity means that: for all $x, y, z \in \Delta$, if $x<_{C_{j}} y$ then $x<_{C_{j}} z$ or $\left.z<_{C_{j}} y\right) ; \sim_{C_{j}}$ is an equivalence relation.

As $\mathcal{E} \mathcal{L}_{\perp}^{+}$has the finite model property (Baader et al. 2005), we can restrict our consideration to interpretations $I$ with a finite domain. In principle, we would like to consider, for each concept $C_{j} \in \mathcal{C}$, all possible domain elements compatible with the inclusions in $\mathcal{T}_{\text {strict }}$, and compare them according to $\leq_{C_{i}}$ relation. This leads us to restrict the 
consideration to models of $\mathcal{T}_{\text {strict }}$ that we call canonical, in analogy with the canonical models of rational closure (Giordano et al. 2013). For each concept $C$ occurring in $K$, let us consider a new concept name $\bar{C}$, (representing the negation of $C$ ) such that $C \sqcap \bar{C} \sqsubseteq \perp$. Let $\mathcal{S}_{\mathcal{K}}$ be the set of all such $C$ and $\bar{C}$, and let $\mathcal{T}_{\text {Constr }}$ the set of all subsumptions $C \sqcap \bar{C} \sqsubseteq \perp$. A set $\left\{D_{1}, \ldots, D_{m}\right\}$ of concepts in $\mathcal{S}_{\mathcal{K}}$ is consistent with $K$ if $\mathcal{T}_{\text {Strict }} \cup \mathcal{T}_{\text {Constr }} \forall_{\mathcal{E L}_{\perp}^{+}} D_{1} \sqcap \cdots \sqcap D_{m} \sqsubseteq \perp$.

\section{Definition 3}

Given a ranked knowledge base $K=\left\langle\mathcal{T}_{\text {strict }}, \mathcal{T}_{C_{1}}, \ldots, \mathcal{T}_{C_{k}}, \mathcal{A}\right\rangle$ an $\mathcal{E} \mathcal{L}_{\perp}^{+}$interpretation $I=$ $\left\langle\Delta,{ }^{I}\right\rangle$ is canonical for $K$ if $I$ satisfies $\mathcal{T}_{\text {strict }}$ and, for any set of concepts $\left\{D_{1}, \ldots, D_{m}\right\} \subseteq$ $\mathcal{S}_{\mathcal{K}}$ consistent with $K$, there exists a domain element $x \in \Delta$ such that, for all $i=1, \ldots, m$, $x \in C^{I}$, if $D_{i}=C$, and $x \notin C^{I}$, if $D_{i}=\bar{C}$.

The idea is that, in a canonical model for $K$, any conjunction of concepts occurring in $K$, or their complements, when consistent with $K$, must have an instance in the domain. Existence of canonical interpretations is guaranteed for knowledge bases which are consistent under the preferential (or ranked) semantics for typicality. $\mathcal{E} \mathcal{L}_{\perp}^{+}$with typicality is indeed a fragment of the description logic $\mathcal{S H \mathcal { I }}$ with typicality, for which existence of canonical models of consistent knowledge bases was proved (Giordano et al. 2018).

In agreement with the preferential interpretations of typicality logics, we further require that, if there is some $C_{h}$-element in a model, then there is at least one $C_{h}$-element satisfying all typicality inclusions for $C_{h}$ (i.e., a prototypical $C_{h}$-element).

\section{Definition 4}

An $\mathcal{E} \mathcal{L}_{\perp}^{+}$interpretation $I=\left\langle\Delta,{ }^{I}\right\rangle$ is $\mathbf{T}$-compliant for $K$ if, I satisfies $\mathcal{T}_{\text {Strict }}$ and, for all $C_{h} \in \mathcal{C}$ such that $C_{h}^{I} \neq \emptyset$, there is some $x \in C_{h}^{I}$ such that $x$ satisfies all defeasible inclusions in $\mathcal{T}_{C_{h}}$.

In a canonical and $\mathbf{T}$-compliant interpretation for $K$, for each $C_{j} \in \mathcal{C}$, the relation $\leq_{C_{j}}$ on the domain $\Delta$ provides a preferential interpretation for the typicality concept $\mathbf{T}\left(C_{j}\right)$ as $\min _{<_{C_{j}}}\left(C_{j}^{I}\right)$, in which all typical $C_{j}$ satisfy all typicality inclusions in $\mathcal{T}_{C_{h}}$.

Existence of a $\mathbf{T}$-compliant canonical interpretation is not guaranteed for an arbitrary knowledge base. For instance, a knowledge base whose typicality inclusions conflict with strict ones (e.g, $\mathbf{T}\left(C_{j}\right) \sqsubseteq D$ and $C_{j} \sqcap D \sqsubseteq \perp$ ) has no $\mathbf{T}$-compliant interpretation. However, existence of $\mathbf{T}$-compliant interpretations is guaranteed for knowledge bases which are consistent under the preferential (or ranked) semantics for typicality (see Appendix A (Giordano and Theseider Dupré 2020), Proposition 5), and consistency can be tested in polynomial time in Datalog (Giordano and Theseider Dupré 2018).

For a ranked knowledge base $K=\left\langle\mathcal{T}_{\text {strict }}, \mathcal{T}_{C_{1}}, \ldots, \mathcal{T}_{C_{k}}, \mathcal{A}\right\rangle$, and a given $\mathcal{E} \mathcal{L}_{\perp}^{+}$interpretation $I=\left\langle\Delta,{ }^{I}\right\rangle$, the strict modular partial order relations $<_{C_{1}}, \ldots,<_{C_{k}}$ over $\Delta$, defined according to Definition 2 above, determine the relative typicality of domain elements w.r.t. each concept $C_{j}$. Clearly, the different preference relations $<_{C_{j}}$ do not need to agree, as seen in the introduction.

\section{Combining multiple preferences into a global preference}

We are interested in defining a notion of typical $C$-element, and defining an interpretation of $\mathbf{T}(C)$, which works for all concepts $C$, not only for the distinguished concepts in $\mathcal{C}$. This 
can be used to evaluate subsumptions of the form $\mathbf{T}(C) \sqsubseteq D$ when $C$ does not belong to $\mathcal{C}$. We address this problem by introducing a notion of multipreference concept-wise interpretation, which generalizes the notion of preferential interpretation (Kraus et al. 1990) by allowing multiple preference relations and, then, combining them in a single (global) preference. Let us consider the following example:

Example 2

Let $K$ be the ranked $\mathrm{KB}\left\langle\mathcal{T}_{\text {strict }}, \mathcal{T}_{\text {Employee }}, \mathcal{T}_{\text {Student }}, \mathcal{T}_{\text {PhDStudent }}, \mathcal{A}\right\rangle$ (with empty $\mathcal{A}=$ $\emptyset)$, containting the strict inclusions:

$\begin{array}{llrl}\text { Employee } \sqsubseteq \text { Adult } & \text { Adult } \sqsubseteq \exists \text { has_SSN. } \top \quad \text { PhdStudent } \sqsubseteq \text { Student } \\ \text { Young } \sqcap \text { NotYoung } \sqsubseteq \perp & & \exists \text { hasScholarship. } \top \sqcap \text { Has_no_Scholarship } \sqsubseteq \perp\end{array}$

The ranked TBox $\mathcal{T}_{\text {Employee }}=\left\{\left(d_{1}, 0\right),\left(d_{2}, 0\right)\right\}$ contains the defeasible inclusions:

$\left(d_{1}\right) \mathbf{T}($ Employee $) \sqsubseteq$ NotYoung $\quad\left(d_{2}\right) \mathbf{T}($ Employee $) \sqsubseteq \exists$ has_boss.Employee

the ranked TBox $\mathcal{T}_{\text {Student }}=\left\{\left(d_{3}, 0\right),\left(d_{4}, 1\right),\left(d_{5}, 1\right)\right\}$ contains the defeasible inclusions:

$\left(d_{3}\right) \mathbf{T}($ Student $) \sqsubseteq \exists$ has_classes. $\top$

$\left(d_{5}\right) \mathbf{T}($ Student $) \sqsubseteq H a s_{-} n o \_S c h o l a r s h i p$

$\left(d_{4}\right) \mathbf{T}($ Student $) \sqsubseteq$ Young

and the ranked TBox $\mathcal{T}_{P h D S t u d e n t}=\left\{\left(d_{6}, 0\right),\left(d_{7}, 1\right)\right\}$ contains the inclusions:

$\left(d_{6}\right) \mathbf{T}($ PhDStudent $) \sqsubseteq \exists$ hasScholarship.Amount

$\left(d_{7}\right) \mathbf{T}($ PhDStudent $) \sqsubseteq$ Bright

We might be interested to check whether typical Italian students are young or whether typical employed students are young. This would require the typicality inclusions $\mathbf{T}($ Student $\sqcap$ Italian $) \sqsubseteq$ Young and $\mathbf{T}($ Employee $\sqcap$ Student $) \sqsubseteq$ Young to be evaluated. Nothing should prevent Italian students from being young (irrelevance). Also, we expect not to conclude that typical employed students are young nor that they are not, as typical students and typical employees have conflicting properties concerning age. However, we would like to conclude that typical employed students have a boss, have classes and have no scholarship, as they should inherit the properties of typical students and of typical employees which are not overridden (i.e., there is no blocking of inheritance). As $\mathrm{PhD}$ students are students, they should inherit all the typical properties of Students, except having no scholarship, which is overridden by $\left(d_{6}\right)$.

To evaluate conditionals $\mathbf{T}(C) \sqsubseteq D$ for any concept $C$ we introduce a concept-wise multipreference interpretation, that combines the preference relations $<_{C_{1}}, \ldots,<_{C_{k}}$ into a single (global) preference relation $<$ and interpreting $\mathbf{T}(C)$ as $(\mathbf{T}(C))^{I}=\min _{<}\left(C^{I}\right)$. The relation $<$ should be defined starting from the preference relations $<_{C_{1}}, \ldots,<_{C_{k}}$ also considering specificity.

Let us consider the simplest notion of specificity among concepts, based on the subsumption hierarchy (one of the notions considered for $\mathcal{D} \mathcal{L}^{N}$ (Bonatti et al. 2015)).

\section{Definition 5 (Specificity)}

Given a ranked $\mathcal{E} \mathcal{L}_{\perp}^{+}$knowledge base $K=\left\langle\mathcal{T}_{\text {strict }}, \mathcal{T}_{C_{1}}, \ldots, \mathcal{T}_{C_{k}}, \mathcal{A}\right\rangle$ over the set of concepts $\mathcal{C}$, and given two concepts $C_{h}, C_{j} \in \mathcal{C}, C_{h}$ is more specific than $C_{j}$ (written $\left.C_{h} \succ C_{j}\right)$ if $\mathcal{T}_{\text {strict }} \models_{\mathcal{E L}_{\perp}^{+}} C_{h} \sqsubseteq C_{j}$ and $\mathcal{T}_{\text {strict }} \not \forall_{\mathcal{E L}_{\perp}^{+}} C_{j} \sqsubseteq C_{h}$.

Relation $\succ$ is irreflexive and transitive (Bonatti et al. 2015). Alternative notions of specificity can be used, based, for instance, on the rational closure ranking.

We are ready to define a notion of multipreference interpretation. Let a relation $<_{C_{i}}$ be well-founded when there is no infinitely-descending chain of domain elements $x_{1}<_{C_{i}}$ $x_{0}, x_{2}<_{C_{i}} x_{1}, x_{3}<_{C_{i}} x_{2}, \ldots$ 
Definition 6 (concept-wise multipreference interpretation)

A (finite) concept-wise multipreference interpretation (or $\mathrm{cw}^{m}$-interpretation) is a tuple $\mathcal{M}=\left\langle\Delta,<_{C_{1}}, \ldots,<_{C_{k}},<,{ }^{I}\right\rangle$ such that: (a) $\Delta$ is a non-empty domain;

(b) for each $i=1, \ldots, k,<_{C_{i}}$ is an irreflexive, transitive, well-founded and modular relation over $\Delta$;

(c) the (global) preference relation $<$ is defined from $<_{C_{1}}, \ldots,<_{C_{k}}$ as follows:

$$
x<y \text { iff } \quad(i) x<_{C_{i}} y \text {, for some } C_{i} \in \mathcal{C}, \text { and }
$$

(ii) for all $C_{j} \in \mathcal{C}, x \leq_{C_{j}} y$ or $\exists C_{h}\left(C_{h} \succ C_{j}\right.$ and $\left.x<_{C_{h}} y\right)$

(d) ${ }^{I}$ is an interpretation function, as defined in $\mathcal{E} \mathcal{L}_{\perp}^{+}$interpretations (see Section 2), with the addition that, for typicality concepts, we let: $(\mathbf{T}(C))^{I}=\min _{<}\left(C^{I}\right)$, where $\operatorname{Min}_{<}(S)=\{u: u \in S$ and $\nexists z \in S$ s.t. $z<u\}$.

Notice that the relation $<$ is defined from $<_{C_{1}}, \ldots,<_{C_{k}}$ based on a modified Pareto condition: $x<y$ holds if there is at least a $C_{i} \in \mathcal{C}$ such that $x<_{C_{i}} y$ and, for all $C_{j} \in \mathcal{C}$, either $x \leq_{C_{j}} y$ holds or, in case it does not, there is some $C_{h}$ more specific than $C_{j}$ such that $x<_{C_{h}} y$ (preference $<_{C_{h}}$ in this case overrides $<_{C_{j}}$ ). For instance, in Example 2, for two domain elements $x, y$, both instances of PhDStudent, Student, $\exists$ has_Classes.T, Young, and such that $x$ is instance of has_no_Scholarship, while $y$ is not, we have that $x<_{\text {Student }} y$ and $y<_{\text {PhDStudent }} x$. As PhDStudent is more specific than Student, globally we get $y<x$. We can prove the following result.

\section{Proposition 1}

Given a $\mathrm{cw}^{m}$-interpretation $\mathcal{M}=\left\langle\Delta,<_{C_{1}}, \ldots,<_{C_{k}},<,{ }^{I}\right\rangle$, relation $<$ is an irreflexive, transitive and well-founded relation.

Proof

Well-foundedness of $<$ is immediate from the restriction to finite models.

To prove irreflexivity and transitivity of $<$, we exploit the fact that each $<_{C_{i}}$ is assumed to be an irreflexive, transitive, well-founded and modular relation on $\Delta$ (see Definition 6 ). Irreflexivity of $<$ follows easily from the irreflexivity of the $<_{C_{h}}$ 's as, for $x<x$ to hold, $x<_{C_{h}} x$ should hold for some $C_{h}$, which is not possible as $<_{C_{h}}$ is irreflexive.

To prove transitivity of $<$, we prove transitivity of $\leq$ defined as follows:

$$
\begin{aligned}
x \leq y \text { iff for all } C_{j} \in \mathcal{C} & (i) x \leq_{C_{j}} y, \text { or } \\
& (\text { ii }) \text { exists } C_{h} \in \mathcal{C},\left(C_{h} \succ C_{j} \text { and } x<_{C_{h}} y\right)
\end{aligned}
$$

It is easy to see that the global preference relation $<$ introduced in point (c) of Definition 6 can be equivalently defined as: $x<y$ iff $(x \leq y$ and not $y \leq x)$. Transitivity of $<$ follows from transitivity of $\leq$.

To prove transitivity of $\leq$, let us assume that $x \leq y$ and $y \leq z$ hold. We prove that $x \leq z$ holds by proving that: for all $C_{j} \in \mathcal{C}, x \leq_{C_{j}} z$ holds (call this case $(i)_{C_{j}}^{x, z}$ ) or there is a $C_{h}$ such that $C_{h} \succ C_{j}$ and $x<_{C_{k}} z$ (call this case $\left.(i i)_{C_{j}}^{x, z}\right)$ ).

As $x \leq y$ holds, for all $C_{j} \in \mathcal{C}, x \leq_{C_{j}} y\left(\right.$ case $\left.(i)_{1}\right)$ or there is a $C_{h}$ such that $C_{h} \succ C_{j}$ and $x<_{C_{k}} y$ (case $\left.(i i)_{1}\right)$. Similarly, as $y \leq z$ holds, for all $C_{j} \in \mathcal{C}, y \leq_{C_{j}} z$ (case $\left.(i)_{2}\right)$ or there is a $C_{r}$ such that $C_{r} \succ C_{j}$ and $x<_{C_{r}} y$ (case $\left.(i i)_{2}\right)$. Let us consider the different possible combination of cases in which $x \leq y$ and $y \leq z$ hold, for each $C_{j}$ : 
Case $(i)_{1^{-}}(i)_{2}$ : In this case, $x \leq_{C_{j}} y$ and $y \leq_{C_{j}} z$ hold. By transitivity of $\leq_{C_{j}}, x \leq_{C_{j}} z$ (i.e., condition $(i)_{C_{j}}^{x, z}$ is satisfied).

Case $(i i)_{1}-(i)_{2}$ : In this case, $y \leq_{C_{j}} z$, and there is a $C_{h}$ such that $C_{h} \succ C_{j}$ and $x<_{C_{h}} y$. Let $C_{h}$ be maximally specific among all concepts $C \in \mathcal{C}$ such that $C \succ C_{j}$ and $x<_{C} y$.

If $y \leq_{C_{h}} z$ is the case, from $x<_{C_{h}} y$, we get $x<_{C_{h}} z$, so that: there is a $C_{h}$ such that $C_{h} \succ C_{j}$ and $x<_{C_{h}} z$, i.e., condition $(i i)_{C_{j}}^{x, z}$ is satisfied. Otherwise, if $z<_{C_{h}} y$, as $y \leq z$, there must be a $C_{r}$ such that $C_{r} \succ C_{h}$ and $y<_{C_{r}} z$. If $x \leq_{C_{r}} y$, we can conclude that $x<_{C_{r}} z$. From $C_{r} \succ C_{h} \succ C_{j}$, by transitivity, $C_{r} \succ C_{j}$, i.e. condition $(i i)_{C_{j}}^{x, z}$ is satisfied. If $x \leq_{C_{r}} y$ does not hold, i.e. $y<_{C_{r}} x$, as $x \leq y$, there must be a $C_{w} \in \mathcal{C}$ such that $C_{w} \succ C_{r}$ and $x<_{C_{w}} y$. However, this is not possible, as it would be $C_{w} \succ C_{r} \succ C_{h} \succ C_{j}$ and we have chosen $C_{h}$ to be maximally specific among the concepts $C \in \mathcal{C}$ such that $C \succ C_{j}$ and $x<_{C} y$, a contradiction.

The cases $(i)_{1}-(i i)_{2}$ and $(i i)_{1}-(i i)_{2}$ can be proved in a similar way.

In a $\mathrm{cw}^{m}$-interpretation we have assumed each $<_{C_{j}}$ to be any irreflexive, transitive, modular and well-founded relation. In a $\mathrm{cw}^{m}$-model of $K$, the preference relations $<_{C_{j}}$ 's will be defined from the ranked TBoxes $\mathcal{T}_{C_{j}}$ 's according to Definition 2.

\section{Definition $7\left(c w^{m}\right.$-model of $\left.K\right)$}

Let $K=\left\langle\mathcal{T}_{\text {strict }}, \mathcal{T}_{C_{1}}, \ldots, \mathcal{T}_{C_{k}}, \mathcal{A}\right\rangle$ be a ranked $\mathcal{E} \mathcal{L}_{\perp}^{+}$knowledge base over $\mathcal{C}$ and $I=\left\langle\Delta,{ }^{I}\right\rangle$ an $\mathcal{E} \mathcal{L}_{\perp}^{+}$interpretation for $K$. A concept-wise multipreference model (or $c w^{m}$-model) of $K$ is a $\mathrm{cw}^{m}$-interpretation $\mathcal{M}=\left\langle\Delta,<_{C_{1}}, \ldots,<_{C_{k}},<,{ }^{I}\right\rangle$ such that: for all $j=1, \ldots, k$, $<_{C_{j}}$ is defined from $\mathcal{T}_{C_{j}}$ and ${ }^{I}$, according to Definition $2 ; \mathcal{M}$ satisfies all strict inclusions inclusions in $\mathcal{T}_{\text {strict }}$ and all assertions in $\mathcal{A}$.

As the preferences $<_{C_{j}}$ 's, defined according to Definition 2, are irreflexive, transitive, wellfounded and modular relations over $\Delta$, a $\mathrm{cw}^{m}$-model $\mathcal{M}$ is indeed a $\mathrm{cw}^{m}$-interpretation. By definition $\mathcal{M}$ satisfies all strict inclusions and assertions in $K$, but is not required to satisfy all typicality inclusions $\mathbf{T}\left(C_{j}\right) \sqsubseteq D$ in $K$, unlike in preferential typicality logics (Giordano et al. 2007).

Consider, in fact, a situation in which typical birds are fliers and typical fliers are birds $(\mathbf{T}(B) \sqsubseteq F$ and $\mathbf{T}(F) \sqsubseteq B)$. In a $\mathrm{cw}^{m}$-model two domain elements $x$ and $y$, which are both birds and fliers, might be incomparable wrt $<$, as $x$ is more typical than $y$ as a bird, while $y$ is more typical than $x$ as a flier, even if one of them is minimal wrt $<_{\text {Bird }}$ and the other is not. In this case, they will be both minimal wrt $<$. In preferential logics, we would conclude that $\mathbf{T}(B) \equiv \mathbf{T}(F)$, which is not the case under the $\mathrm{cw}^{m}$-semantics. This implies that the notion of $\mathrm{cw}^{m}$-entailment (defined below) is not stronger than preferential entailment. It is also not weaker as, for instance, in Example 2, $\mathrm{cw}^{m}$-entailment allows to conclude that typical employed students have a boss, have classes and no scholarship (although defaults $\left(d_{1}\right)$ and $\left(d_{4}\right)$ are conflicting), while neither preferential entailment nor the rational closure would allow such conclusions; $\mathrm{cw}^{m}$-entailment does not suffer from inheritance blocking, and is then incomparable with preferential entailment and with entailment under rational closure, being neither weaker nor stronger.

The notion of $\mathrm{cw}^{m}$-entailment exploits canonical and T-compliant $\mathrm{cw}^{m}$-models of $K$. A cw ${ }^{m}$-model $\mathcal{M}=\left\langle\Delta,<_{C_{1}}, \ldots,<_{C_{k}},<,{ }^{I}\right\rangle$ is canonical ( $\mathbf{T}$-compliant) for $K$ if the $\mathcal{E} \mathcal{L}_{\perp}^{+}$ interpretation $\left\langle\Delta,{ }^{I}\right\rangle$ is canonical (T-compliant) for $K$. 
Definition $8\left(c w^{m}\right.$-entailment)

An inclusion $\mathbf{T}(C) \sqsubseteq C_{j}$ is $\mathrm{cw}^{m}$-entailed from $K$ (written $K \models_{c w^{m}} \mathbf{T}(C) \sqsubseteq C_{j}$ ) if $\mathbf{T}(C) \sqsubseteq C_{j}$ is satisfied in all canonical and $\mathbf{T}$-compliant $\mathrm{cw}^{m}$-models $\mathcal{M}$ of $K$.

It can be proved that $\mathrm{cw}^{m}$-entailment satisfies the KLM postulates of a preferential consequence relation (Proposition 6, Appendix A (Giordano and Theseider Dupré 2020)).

\section{Reasoning under the cw-multipreference semantics}

In this section we consider the problem of checking $\mathrm{cw}^{m}$-entailment of a typicality subsumption $\mathbf{T}(C) \sqsubseteq D$ as a problem of determining preferred answer sets. Based on this formulation, that we prove to be sound and complete, we show that the problem is in $\Pi_{2}^{p}$. We exploit asprin (Brewka et al. 2015) to compute preferred answer sets. The proofs for this section can be found in Appendix C (Giordano and Theseider Dupré 2020).

In principle, for checking $\mathbf{T}(C) \sqsubseteq D$ we would need to consider all possible typical $C$ elements in all possible canonical and $\mathbf{T}$-compliant $\mathrm{cw}^{m}$-model of $K$, and verify whether they are all instances of $D$. However, we will prove that it is sufficient to consider, among all the (finite) $\mathrm{cw}^{m}$-models of $K$, the polynomial $\mathcal{E} \mathcal{L}_{\perp}^{+}$models that we can construct using the $\mathcal{E} \mathcal{L}_{\perp}^{+}$fragment of the materialization calculus for $\mathcal{S} \mathcal{R O} \mathcal{E} \mathcal{L}(\sqcap, \times)$ (Krötzsch 2010), by considering all alternative interpretations for a distinguished element aux $x_{C}$, representing a prototypical $C$-element. In preferred models, which minimize the violation of typicality inclusions by $a u x_{C}$, it indeed represents a typical $C$-element. An interesting result is that neither we need to consider all the possible interpretations for constants in the model nor to minimize violation of typicalities for them. Essentially, when evaluating the properties of typical employed students we are not concerned with the typicality (or atypicality) of other constants in the model (e.g., with typical cars, with typical birds, and with typical named individuals). Unlike a previous semantics by Giordano and Theseider Dupré (2016), which generalizes rational closure by allowing typicality concepts on the rhs of inclusions, we are not required to consider all possible alternative interpretations and ranks of individuals in the model. We will see, however, that we do not loose solutions (models) in this way.

In the following we first describe how answer sets of a base program, corresponding to $\mathrm{cw}^{m}$-models of $K$, are generated. Then we describe how preferred models can be selected, where $a u x_{C}$ represent a typical $C$-element.

We will assume that assertions $(C(a)$ and $r(a, b))$ are represented using nominals as inclusions (resp., $\{a\} \sqsubseteq A$ and $\{a\} \sqsubseteq \exists R .\{b\}$ ), where a nominal $\{a\}$ is a concept containing a single element and $(\{a\})^{I}=\left\{a^{I}\right\}$. We also assume that the knowledge base $K$ is in normal form (Baader et al. 2005), where a typicality inclusion $\mathbf{T}(B) \sqsubseteq C$ is in normal form when $B, C \in N_{C}$ (Giordano and Theseider Dupré 2016). Extending the results in (Krötzsch 2010), it can be proved that, given a KB, a semantically equivalent $\mathrm{KB}$ in normal form (over an extended signature) can be computed in linear time. We refer to a previous paper (Giordano and Theseider Dupré 2018) for the details on normalization.

The base program $\Pi(K, C, D)$ for the (normalized) knowledge base $K$ and typicality subsumption $\mathbf{T}(C) \sqsubseteq D$ is composed of three parts, $\Pi(K, C, D)=\Pi_{K} \cup \Pi_{I R} \cup \Pi_{C, D}$.

$\Pi_{K}$ is the representation of $K$ in Datalog (Krötzsch 2010), where, to keep a DL-like notation, we do not follow the convention where variable names start with uppercase; in 
particular, $A, C, D$ and $R$, are intended as ASP constants corresponding to the same class/role names in $K$. In this representation, nom $(a), \operatorname{cls}(A), \operatorname{rol}(R)$ are used for $a \in N_{I}$, $A \in N_{C}, R \in N_{R}$, and, for example, subClass $(a, C)$, subClass $(A, C)$ are used for $C(a)$, $A \sqsubseteq C$. Additionally, $\operatorname{subTyp}(C, D, N)$ is used for $T(C) \sqsubseteq D$ having rank $N$, and the following definitions for distinguished concepts, typical properties, and valid ranks, will be used in defining preferences:

$$
\begin{aligned}
& \operatorname{dcls}(C) \leftarrow \operatorname{subTyp}(C, D, N) \\
& \operatorname{tprop}(C, D) \leftarrow \operatorname{subTyp}(C, D, N) \\
& \operatorname{validrank}(C, N) \leftarrow \operatorname{subTyp}(C, D, N)
\end{aligned}
$$

For each distinguished concept $C_{i}$, auxtc $\left(a u x_{-} C i, C i\right)$ is included, where $a u x_{-} C i$ is an auxiliary individual name. Other auxiliary constants (one for each inclusion $A \sqsubseteq \exists R . B$ ) are needed (Krötzsch 2010) to deal with existential rules.

$\Pi_{I R}$ contains the subset of the inference rules (1-29) for instance checking (Krötzsch 2010 ) that is relevant for $\mathcal{E} \mathcal{L}_{\perp}^{+}$(reported in Appendix B (Giordano and Theseider Dupré 2020)), for example inst $(x, z) \leftarrow \operatorname{subClass}(y, z)$, inst $(x, y)$; for $\perp$, an additional rule is used: $\leftarrow \operatorname{bot}(z), \operatorname{inst}(x, z)$. Additionally, $\Pi_{I R}$ contains the version of the same rules for subclass checking (where inst_sc $(A, B, A)$ represents $A \sqsubseteq B$ (Krötzsch 2010)), and then the following rule encodes specificity $C_{h} \succ C_{j}$ :

morespec $(C h, C j) \leftarrow d c l s(C h), d c l s(C j)$, inst_sc $(C h, C j, C h)$, not inst_sc $(C j, C h, C j)$

$\Pi_{I R}$ also contains the following rules:

(a) $\left\{\operatorname{inst}\left(\operatorname{aux}_{C}, D\right)\right\} \leftarrow d c l s(C i), \operatorname{inst}\left(\operatorname{aux}_{C}, C i\right), \operatorname{tprop}(C i, D)$

(b) $\operatorname{inst}(Y, C i) \leftarrow \operatorname{auxtc}(Y, C i)$, inst $(X, C i)$

(c) $\operatorname{typ}(Y, C i) \leftarrow \operatorname{auxtc}(Y, C i), \operatorname{inst}(Y, C i)$

(d) $\operatorname{inst}(Y, D) \leftarrow \operatorname{subTyp}(C i, D, N), \operatorname{typ}(Y, C i)$

Rule (a) generates alternative answer sets (corresponding to different interpretations) where $a u x_{C}$ may have the typical properties of the concepts it belongs. The constant $a u x_{-} C i$, such that auxtc(aux_Ci,Ci) holds, represents a typical $C i$ (a minimal element wrt. $\leq_{C_{i}}$ ) only in case it is an instance of $C_{i}$ (i.e., inst (aux_Ci, $C i$ ) holds). Rule (b) establishes that, if there is an instance $x$ of concept $C_{i}$ in the interpretation, then $a u x_{-} C i$ must be an instance of $C_{i}$ (it models $\mathbf{T}$-compliance) and, by rule (c), aux_Ci is a typical instance of $C_{i}$, i.e., it is minimal wrt. $\leq_{C_{i}}$ among $C_{i}$-elements in the interpretation at hand. By rule (d), a typical instance of $C_{i}$ has all typical properties of $C_{i}$. The rules (b)-(d) only allow to derive conclusions involving aux_Ci constants.

$\Pi_{C, D}$ contains (if necessary) normalized axioms defining $C, D$ in $\mathbf{T}(C) \sqsubseteq D$ in terms of other concepts (e.g., replacing $\mathbf{T}($ Employee $\sqcap$ Student $) \sqsubseteq$ Young with $\mathbf{T}(A) \sqsubseteq$ Young and $A \sqsubseteq$ Employee, $A \sqsubseteq$ Student and Employee $\sqcap$ Student $\sqsubseteq A)$ plus the facts auxtc $\left(\operatorname{aux}_{C}, C\right)$, $\operatorname{nom}\left(\operatorname{aux}_{C}\right)$, inst $\left(\operatorname{aux}_{C}, C\right)$.

Proposition 2

Given a normalized ranked knowledge base $K=\left\langle\mathcal{T}_{\text {strict }}, \mathcal{T}_{C_{1}}, \ldots, \mathcal{T}_{C_{k}}, \mathcal{A}\right\rangle$ over the set of concepts $\mathcal{C}$, and a (normalized) subsumption $C \sqsubseteq D$ :

(1) if there is an answer set $S$ of the ASP program $\Pi(K, C, D)$, such that inst $\left(\right.$ aux $\left._{C}, D\right) \notin$ $S$, then there is a $\mathbf{T}$-compliant $\mathrm{cw}^{m}$-model $\mathcal{M}=\left\langle\Delta,<_{C_{1}}, \ldots,<_{C_{k}},<,{ }^{I}\right\rangle$ for $K$ that falsifies the subsumption $C \sqsubseteq D$. 
(2) if there is a $\mathbf{T}$-compliant $\mathrm{cw}^{m}$-model $\mathcal{M}=\left\langle\Delta,<_{C_{1}}, \ldots,<_{C_{k}},<,{ }^{I}\right\rangle$ of $K$ that falsifies the subsumption $C \sqsubseteq D$, then there is an answer set $S$ of $\Pi(K, C, D)$, such that $\operatorname{inst}\left(\operatorname{aux}_{C}, D\right) \notin S$.

We exploit the idea of identifying the minimal $C$-elements in a canonical $\mathrm{cw}^{m}$-model of $K$, as the aux $_{C}$ elements of the preferred answer sets of $\Pi(K, C, D)$.

\section{Definition 9}

Let $S$ and $S^{\prime}$ be answer sets of $\Pi(K, C, D)$. $S^{\prime}$ is preferred to $S$ if aux $x_{C}$ in $S^{\prime}$ (denoted as $a u x_{C}^{S^{\prime}}$ ) is globally preferred to $a u x_{C}$ in $S$ (denoted as $a u x_{C}^{S}$ ), that is, $a u x_{C}^{S^{\prime}}<a u x_{C}^{S}$, defined according to Definition 6, point (c), provided that relations aux $x_{C}^{S^{\prime}} \leq_{C_{j}} a u x_{C}^{S}$ are defined according to Definition 2, by letting:

$$
\mathcal{T}_{C_{i}}^{l}\left(a u x_{C}^{S}\right)=\left\{B \mid \operatorname{inst}\left(a u x_{C}, C_{i}\right) \notin S \text { or } \operatorname{inst}\left(a u x_{C}, B\right) \in S, \text { for } \mathbf{T}\left(C_{i}\right) \sqsubseteq B \in K\right\},
$$

i.e., $\mathcal{T}_{C_{i}}^{l}\left(a u x_{C}^{S}\right)$ contains the $B$ 's such that $C_{i} \sqsubseteq B$ is satisfied in $S$ for some typicality inclusion $\mathbf{T}\left(C_{i}\right) \sqsubseteq B$ in $K$; and similarly for $S^{\prime}$. The strict relation $a u x_{C}^{S^{\prime}}<_{C_{j}}$ aux $x_{C}^{S}$ is defined accordingly.

Essentially, we compare $S$ and $S^{\prime}$ identifying the concepts of which aux $x_{C}$ is an instance in $S$ and in $S^{\prime}$ and evaluating which defaults are satisfied for aux $x_{C}$ in $S$ and in $S^{\prime}$, using the same criteria used for comparing domain elements in Section 3.

The selection of preferred answer sets, the ones where $a u x_{C}$ is in $\min _{<}\left(C^{I}\right)$, and then in $(\mathbf{T}(C))^{I}$, can be done in asprin with the following preference specification:

$$
\begin{aligned}
& \text { \#preference }(p, \text { multipref })\{d \operatorname{dcls}(C i): \operatorname{dcls}(C i) ; \text { morespec }(C i, C j): \operatorname{dcls}(C i), \operatorname{dcls}(C j) ; \\
& \quad \operatorname{inst}(\operatorname{aux} C, E): \operatorname{tprop}(C i, E), d \operatorname{dcl}(C i) ; \operatorname{subTyp}(C i, E, R): \operatorname{subTyp}(C i, E, R) ; \\
& \operatorname{validrank}(C i, R): \operatorname{validrank}(C i, R)\}
\end{aligned}
$$

\#optimize $(p)$

requiring optimization wrt $p$ which is a preference of type multipref, a preference type defined by the preference program below (exploiting the fact that asprin, among other things, generates from the specification a fact preference ( $p$, multipref $)$ ).

In asprin preference programs, defining whether an answer set $S$ is preferred to $S^{\prime}$ according to a preference $P$ amounts to defining a predicate $\operatorname{better}(P)$ for the case where $P$ is of the type being defined; the predicates holds and holds' are used to check whether the atoms in the preference specification hold in $S$ and $S^{\prime}$, respectively. In the following, better $(p)$, bettereqwrt ( $C i)$, betterwrt $(C i)$, correspond to $<, \leq_{C_{i}},<_{C_{i}}$, respectively, for $a u x_{C}^{S}$ and $a u x_{C}^{S^{\prime}}$, comparing what holds for aux $x_{C}$ to what holds' for it; moreprop and samenumprop verify whether more (or the same number of ) typicality inclusions of rank $R$ are satisfied by aux $_{C}$ in $S$ wrt $S^{\prime}$ :

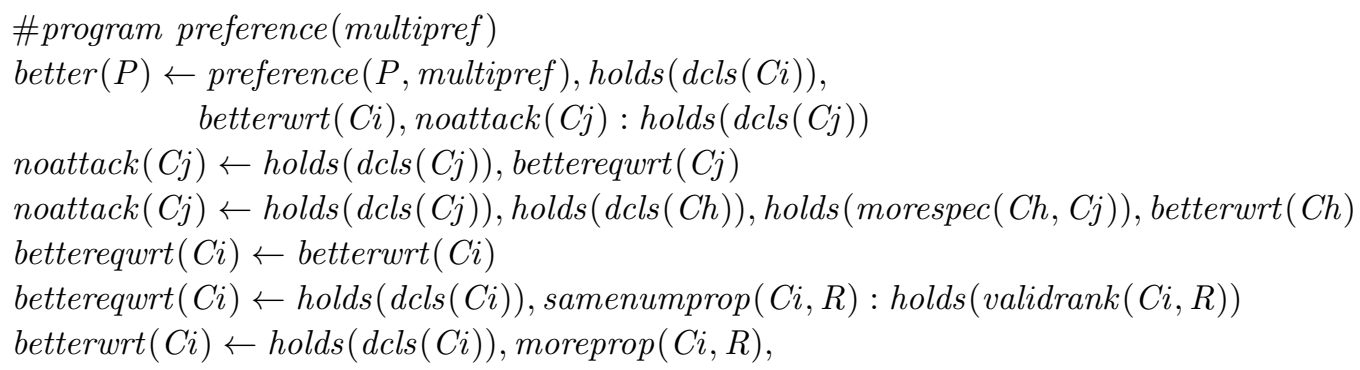




$$
\begin{aligned}
& \text { samenumprop }(\mathrm{Ci}, \mathrm{R} 1) \text { : holds (validrank }(\mathrm{Ci}, \mathrm{R} 1)), R 1>R \\
& \text { moreprop }(\mathrm{Ci}, \mathrm{R}) \leftarrow \text { holds }(\operatorname{validrank}(\mathrm{Ci}, \mathrm{R})) \text {, } \\
& \text { \#sum }\{-1, E: \operatorname{sat}(\operatorname{aux} C, C i, E), \operatorname{holds}(\operatorname{subTyp}(C i, E, R)) \text {; } \\
& \text { 1, E : sat1 }(\operatorname{aux} C, C i, E), \operatorname{holds}(\operatorname{subTyp}(C i, E, R))\}-1 \\
& \operatorname{sat}(\operatorname{aux} C, C i, E) \leftarrow \operatorname{holds}(X), X=\operatorname{inst}(\operatorname{aux} C, E), \operatorname{holds}(\operatorname{subTyp}(C i, E, R)) \\
& \operatorname{sat}(\operatorname{aux} C, C i, E) \leftarrow \text { not holds }(X), X=\operatorname{inst}(\operatorname{aux} C, C i), \operatorname{holds}(\operatorname{subTyp}(C i, E, R)) \\
& \operatorname{sat1}(\operatorname{aux} C, C i, E) \leftarrow \operatorname{holds}^{\prime}(X), X=\operatorname{inst}(\operatorname{aux} C, E), \operatorname{holds}(\operatorname{subTyp}(C i, E, R)) \\
& \operatorname{sat1}(\operatorname{aux} C, C i, E) \leftarrow \text { not holds }(X), X=\operatorname{inst}(\operatorname{aux} C, C i), \operatorname{holds}(\operatorname{subTyp}(C i, E, R)) \\
& \text { samenumprop }(\mathrm{Ci}, \mathrm{R}) \leftarrow \text { holds }(\operatorname{validrank}(\mathrm{Ci}, \mathrm{R})) \text {, } \\
& 0 \# \operatorname{sum}\{-1, E \text { : } \operatorname{sat}(\operatorname{aux} C, C i, E), \operatorname{holds}(\operatorname{subTyp}(C i, E, R)) \text {; } \\
& \text { 1, E : sat1 (auxC,Ci,E), holds }(\operatorname{subTyp}(C i, E, R))\} 0
\end{aligned}
$$

Let us call Pref the preference specification and the preference program defined above; checking whether $\mathbf{T}(C) \sqsubseteq D$ is $\mathrm{cw}^{m}$-entailed amounts to checking whether $\operatorname{inst}\left(\operatorname{aux}_{C}, D\right)$ is in all preferred answer sets of $\Pi(K, C, D)$ according to Pref.

\section{Proposition 3}

Given a normalized ranked knowledge base $K=\left\langle\mathcal{T}_{\text {strict }}, \mathcal{T}_{C_{1}}, \ldots, \mathcal{T}_{C_{k}}, \mathcal{A}\right\rangle$ over the set of concepts $\mathcal{C}$, and a subsumption $\mathbf{T}(C) \sqsubseteq D$, we can prove the following:

(1) if there is a canonical and T-compliant $\mathrm{cw}^{m}$-model $\mathcal{M}=\left(\Delta,<_{C_{1}}, \ldots,<_{C_{k}},<, \cdot^{I}\right)$ of $K$ that falsifies $\mathbf{T}(C) \sqsubseteq D$, then there is a preferred answer set $S$ of $\Pi(K, C, D)$ according to Pref, such that inst $\left(\operatorname{aux}_{C}, D\right) \notin S$.

(2) if there is a preferred answer set $S$ of $\Pi(K, C, D)$ according to Pref, such that $\operatorname{inst}\left(\operatorname{aux}_{C}, D\right) \notin S$, then there is a canonical and $\mathbf{T}$-compliant $\mathrm{cw}^{m}$-model $\mathcal{M}=$ $\left(\Delta,<_{C_{1}}, \ldots,<_{C_{k}},<,^{I}\right)$ of $K$ that falsifies $\mathbf{T}(C) \sqsubseteq D$.

Propositions 2 and 3 tell us that, for computing $\mathrm{cw}^{m}$-entailment, it is sufficient to consider the polynomial T-compliant $\mathrm{cw}^{m}$-models of $K$ corresponding to answer sets $S$ of $\Pi(K$, $C, D)^{1}$. A $\Pi_{2}^{p}$ upper bound on the complexity of $\mathrm{cw}^{m}$-entailment can be proved based on the the above formulation of $\mathrm{cw}^{m}$-entailment as a problem of computing preferred answer sets. The $\Pi_{2}^{p}$-hardness can be proved by providing a reduction of the minimal entailment problem of positive disjunctive logic programs, which was proved to be a $\Pi_{2}^{P}$-hard problem by Eiter and Gottlob (1995).

Proposition 4

Deciding $\mathrm{cw}^{m}$-entailment is a $\Pi_{2}^{p}$-complete problem.

\subsection{Some experimental results}

For Example 2, we actually get that typical employed students have a boss, but not that they are young: there are, in fact, two preferred answer sets, with inst (aux $\left.x_{C}, Y_{\text {oung }}\right)$ and inst $\left(\right.$ aux $_{C}$, NotYoung) respectively; they are generated in 0.40 seconds.

A first scalability test is based on a slightly larger version of the same example, with 5 distinguished classes and 50 typicality inclusions. Adding to it more typicality inclusions,

1 Note that, for verifying $\mathrm{cw}^{m}$-entailment of $\mathbf{T}(C) \sqsubseteq D$, all answer sets of $\Pi(K, C, D)$ have to be considered and checking whether $\Pi(K, C, D) \cup\left\{-i n s t\left(\operatorname{aux}_{C}, D\right)\right\}$ has no preferred answer sets would not be correct. 


\begin{tabular}{|c|c|c|c|c|c|}
\hline & $\|$ & $1 \mathrm{x}$ & $2 \mathrm{x}$ & $4 \mathrm{x}$ & $8 \mathrm{x}$ \\
\hline test $1 \mathrm{a}$ & $\|$ & 0.35 & 0.45 & 0.63 & 0.99 \\
\hline test $1 b$ & $\|$ & 0.35 & 0.50 & 0.95 & 3.93 \\
\hline test 2 & $\|$ & 1.03 & 1.15 & 1.27 & 1.76 \\
\hline
\end{tabular}

Table 1. Some scalability results

up to 8 times (400 inclusions), the runtime grows up to $0.99 \mathrm{~s}$ (see Table 1 , test 1a, average running times for asprin 1.1.1 under Linux on an Intel Xeon E5-2640 @ 2.00GHz). Adding up to 8 copies of the KB (i.e., adding $\mathbf{T}\left(\right.$ Employee $\left.^{\prime}\right) \sqsubseteq$ Not Young ${ }^{\prime}$ and similar), with up to 40 distinguished classes and 400 typicality inclusions, the runtime grows up to 3.93 (Table 1 , test $1 \mathrm{~b})$.

In another experiment, we have distinguished classes $C_{1} \ldots C_{5}$ with $C_{3} \sqsubseteq C_{2} \sqsubseteq C_{1}$, $C_{5} \sqsubseteq C_{4} \sqsubseteq C_{1}$. For all $i$, the $C_{i}$ 's are typically $P_{i}$ 's, $Q_{i}$ 's, $R_{i}$ 's, where for $i \neq j, P_{i} \sqcap P_{j} \sqsubseteq \perp$. A typical $C_{3} \sqcap C_{5}$ then inherits all the $Q_{i}$ 's and $R_{i}$ 's properties, while it can either be a $P_{3}$ or a $P_{5}$. Also in this case adding up to 8 copies of the $\mathrm{KB}$ (with then up to 40 distinguished classes and 120 typicality inclusions) leads to a moderate increase of the running time which ranges from 1.03 to 1.76 seconds (Table 1, test 2).

Dealing with longer chains of subclasses seems more challenging. For a modification of the base case of the previous example with 10 distinguished classes $C_{10} \sqsubseteq C_{8} \sqsubseteq C_{6} \sqsubseteq$ $C_{4} \sqsubseteq C_{2} \sqsubseteq C_{1}, C_{9} \sqsubseteq C_{7} \sqsubseteq C_{5} \sqsubseteq C_{3} \sqsubseteq C_{1}$, and 50 typicality axioms, checking the properties of typical $C_{9} \sqcap C_{10}$ already takes 5.4 seconds.

\section{Conclusions and related work}

In this paper we have developed an ASP approach for defeasible inference in a conceptwise multipreference extension of $\mathcal{E} \mathcal{L}^{\perp}$. Our semantics is related to the multipreference semantics for $\mathcal{A L C}$ developed by Gliozzi (2016), which is based on the idea of refining the rational closure construction considering the preference relations $<_{A_{i}}$ associated with different aspects, but we follow a different route concerning both the definition of the preference relations associated with concepts, and the way of combining them in a single preference relation. In particular, Gliozzi's multipreference semantics aims at defining a refinement of rational closure semantics, which is not our aim here; compared with rational closure, our semantics is neither weaker (as it does not suffer from the "the blocking of property inheritance" problem) nor stronger (see Section 4).

The idea of having different preference relations, associated with different typicality operators, has been studied by Gil (2014) to define a multipreference formulation of the description logic $\mathcal{A L C}+\mathbf{T}_{m i n}$, a typicality DL with a minimal model preferential semantics. In this proposal we associate preferences with concepts, and we combine such preferences into a single global one. For a preferential extension of $\mathcal{E} \mathcal{L}^{\perp}$ based on the same minimal model semantics as $\mathcal{A L C}+\mathbf{T}_{\text {min }}$, it has been proved (Giordano et al. 
2011) that minimal entailment is already ExPTIME-hard for $\mathcal{E} \mathcal{L}^{\perp} \mathrm{KBs}$, while a $\Pi_{2}^{p}$ upper bound holds for minimal entailment in the Left Local fragment of $\mathcal{E} \mathcal{L}^{\perp} \mathbf{T}_{\text {min }}$, as for circumscriptive KBs (Bonatti et al. 2011). A related problem of commonsense concept combination has been addressed in a probabilistic extension of $\mathcal{A L C}+\mathbf{T}_{\mathrm{R}}$ (Lieto and Pozzato 2018).

Among the formalisms combining DLs with logic programming rules (Eiter et al. 2008; Eiter et al. 2011; Motik and Rosati 2010; Knorr et al. 2012; Gottlob et al. 2014) DLprograms (Eiter et al. 2008; Eiter et al. 2011) support a loose coupling of DL ontologies and rule-based reasoning under the answer set semantics and the well-founded semantics; rules may contain DL-atoms in their bodies, corresponding to queries to a DL ontology, which can be modified according to a list of updates. The non-monotonic description logic $\mathcal{D} \mathcal{L}^{N}$ (Bonatti et al. 2015) supports normality concepts based on a notion of overriding, enjoying good computational properties, and preserves the tractability for low complexity DLs, including $\mathcal{E} \mathcal{L}^{\perp^{++}}$and DL-lite (Bonatti et al. 2015). Bozzato et al. (2014; 2018) present extensions of the CKR (Contextualized Knowledge Repositories) framework in which defeasible axioms are allowed in the global context and exceptions can be handled by overriding and have to be justified in terms of semantic consequence. A translation of extended CKRs (with knowledge bases in $\mathcal{S} \mathcal{R O} \mathcal{I} \mathcal{Q}$-RL) into Datalog programs under the answer set semantics is developed. Related approaches are also the work by Beierle et al. (2018), characterizing skeptical c-inference as a constraint satisfaction problem, and the work by Deane et al. (2015) presenting an inconsistency tolerant semantics for $\mathcal{A L C}$ using preference weights and exploiting ASP optimization for computing preferred interpretations. Reasoning under the rational closure for low complexity DLs has been investigated for $\mathcal{S} \mathcal{R O} \mathcal{E} \mathcal{L}(\sqcap, \times)$ (Giordano and Theseider Dupré 2018), using a Datalog plus stratified negation polynomial construction and for $\mathcal{E} \mathcal{L} \mathcal{O}_{\perp}$ (Casini et al. 2019), developing a polynomial time subsumption algorithm for the nominal safe fragment (Kazakov et al. 2014). A problem that we have not considered in this paper is the treatment of defeasible information for existential concepts; it has been addressed by Pensel and Turhan (2018), who developed a stronger version of rational and relevant entailment in $\mathcal{E} \mathcal{L}^{\perp}$, exploiting a materialisation-based algorithm for $\mathcal{E} \mathcal{L}^{\perp}$ and a canonical model construction.

It is known that Brewka's \# strategy (2004) exploits the lexicographical order also used by Lehmann to define the models of the lexicographic closure of a conditional knowledge base (Lehmann 1995), starting from the rational closure ranking. This suggests that, while we have used this strategy for ranked TBox $\mathcal{T}_{C_{j}}$ containing only typicality inclusions of the form $\mathbf{T}\left(C_{j}\right) \sqsubseteq D$, coarsely grained ranked TBoxes could be allowed, in which $\mathcal{T}_{C_{j}}$ contains all typicality inclusions $\mathbf{T}(E) \sqsubseteq D$ for any subclass $E$ of $C_{j}$. We expect that this might improve performances, by reducing the number of $\leq_{C_{j}}$ relations to be considered. We leave for future work investigating whether our ASP approach with preferences can be used for computing the lexicographic closure for $\mathcal{E} \mathcal{L}_{\perp}^{+}$, and whether alternative notions of specificity can be adopted.

The modular separation of the typicality inclusions in different TBoxes and their separate use for defining preferences $\leq_{C_{i}}$ suggests that some of the optimizations used by ELK reasoning algorithms (Kazakov et al. 2014) might be extended to our setting.

Acknowledgement: We thank the anonymous referees for their helpful comments and suggestions. This research is partially supported by INDAM-GNCS Project 2019. 


\section{References}

BaAder, F., Brandt, S., And Lutz, C. 2005. Pushing the $\mathcal{E} \mathcal{L}$ envelope. In Proceedings of the 19th International Joint Conference on Artificial Intelligence (IJCAI 2005), L. Kaelbling and A. Saffiotti, Eds. Professional Book Center, Edinburgh, Scotland, UK, 364-369.

BaAder, F. And Hollunder, B. 1995. Embedding defaults into terminological knowledge representation formalisms. J. Autom. Reasoning 14, 1, 149-180.

Beierle, C., Eichhorn, C., Kern-Isberner, G., And Kutsch, S. 2018. Properties of skeptical c-inference for conditional knowledge bases and its realization as a constraint satisfaction problem. Ann. Math. Artif. Intell. 83, 3-4, 247-275.

Bonatti, P. A. 2019. Rational closure for all description logics. Artif. Intell. 274, 197-223.

Bonatti, P. A., Faella, M., Petrova, I., and Sauro, L. 2015. A new semantics for overriding in description logics. Artif. Intell. 222, 1-48.

Bonatti, P. A., Faella, M., And Sauro, L. 2011. Defeasible inclusions in low-complexity dls. J. Artif. Intell. Res. (JAIR) 42, 719-764.

Bonatti, P. A., Lutz, C., And Wolter, F. 2009. The Complexity of Circumscription in DLs. Journal of Artificial Intelligence Research (JAIR) 35, 717-773.

Bonatti, P. A., Petrova, I. M., And Sauro, L. 2015. Optimizing the computation of overriding. In Proc. ISWC 2015. 356-372.

Bozzato, L., Eiter, T., And Serafini, L. 2014. Contextualized knowledge repositories with justifiable exceptions. In $D L$ 2014. CEUR Workshop Proceedings, vol. 1193. 112-123.

Bozzato, L., Eiter, T., And Serafini, L. 2018. Enhancing context knowledge repositories with justifiable exceptions. Artif. Intell. 257, 72-126.

Brewka, G. 1989. Preferred subtheories: An extended logical framework for default reasoning. In Proceedings of the 11th International Joint Conference on Artificial Intelligence. Detroit, MI, USA, August 1989. 1043-1048.

BrewkA, G. 2004. A rank based description language for qualitative preferences. In Proceedings of the 16th Eureopean Conference on Artificial Intelligence, ECAI'2004, Valencia, Spain, August 22-27, 2004. 303-307.

Brewka, G., Delgrande, J. P., Romero, J., And Schaub, T. 2015. asprin: Customizing answer set preferences without a headache. In Proc. AAAI 2015. 1467-1474.

Britz, K., Heidema, J., And Meyer, T. 2008. Semantic preferential subsumption. In Principles of Knowledge Representation and Reasoning: Proceedings of the 11th International Conference (KR 2008), G. Brewka and J. Lang, Eds. AAAI Press, Sidney, Australia, 476-484.

Casini, G., Meyer, T., Varzinczak, I. J., , And Moodley, K. 2013. Nonmonotonic Reasoning in Description Logics: Rational Closure for the ABox. In 26th International Workshop on Description Logics (DL 2013). CEUR Workshop Proceedings, vol. 1014. 600-615.

Casini, G. and Straccia, U. 2010. Rational Closure for Defeasible Description Logics. In Proc. 12th European Conf. on Logics in Artificial Intelligence (JELIA 2010), T. Janhunen and I. Niemelä, Eds. LNCS, vol. 6341. Springer, Helsinki, Finland, 77-90.

Casini, G. And Straccia, U. 2013. Defeasible inheritance-based description logics. Journal of Artificial Intelligence Research (JAIR) 48, 415-473.

Casini, G., Straccia, U., and Meyer, T. 2019. A polynomial time subsumption algorithm for nominal safe elo $\perp$ under rational closure. Inf. Sci. 501, 588-620.

Deane, G., Broda, K., And Russo, A. 2015. Reasoning in the presence of inconsistency through preferential ALC. In 20th International Conferences on Logic for Programming, Artificial Intelligence and Reasoning, LPAR 2015, Suva, Fiji, November 24-28, 2015. 67-80.

Donini, F. M., Nardi, D., And Rosati, R. 2002. Description logics of minimal knowledge and negation as failure. ACM Transactions on Computational Logic (ToCL) 3, 2, 177-225.

Eiter, T. And Gottlob, G. 1995. On the computational cost of disjunctive logic programming: Propositional case. Annals of Mathematics and Artificial Intelligence 15, 3-4, 289-323. 
Eiter, T., Ianni, G., Lukasiewicz, T., and Schindlauer, R. 2011. Well-founded semantics for description logic programs in the semantic web. ACM Trans. Comput. Log. 12, 2, 11.

Eiter, T., Ianni, G., Lukasiewicz, T., Schindlauer, R., and Tompits, H. 2008. Combining answer set programming with description logics for the semantic web. Artif. Intell. 172, 12-13, 1495-1539.

GIL, O. F. 2014. On the Non-Monotonic Description Logic ALC+T $\min$. CoRR abs/1404.6566.

Giordano, L., Gliozzi, V., And Olivetti, N. 2018. Towards a rational closure for expressive description logics: the case of SHIQ. Fundam. Inform. 159, 1-2, 95-122.

Giordano, L., Gliozzi, V., Olivetti, N., and Pozzato, G. 2013. Minimal Model Semantics and Rational Closure in Description Logics . In 26th International Workshop on Description Logics (DL 2013). Vol. 1014. 168 - 180.

Giordano, L., Gliozzi, V., Olivetti, N., and Pozzato, G. L. 2007. Preferential Description Logics. In Proc. LPAR 2007 (14th Conf. on Logic for Programming, Artificial Intelligence, and Reasoning), N. Dershowitz and A. Voronkov, Eds. LNAI, vol. 4790. Springer-Verlag, 257-272.

Giordano, L., Gliozzi, V., Olivetti, N., and Pozzato, G. L. 2011. Reasoning about typicality in low complexity DLs: the logics $\mathcal{E} \mathcal{L}^{\perp} \mathbf{T}_{\text {min }}$ and DL-Lite $\mathbf{T}_{\text {min }}$. In Proc. 22nd Int. Joint Conf. on Artificial Intelligence (IJCAI 2011). Morgan Kaufmann, Barcelona, 894-899.

Giordano, L. And Theseider Dupré, D. 2016. ASP for minimal entailment in a rational extension of SROEL. TPLP 16, 5-6, 738-754.

Giordano, L. And Theseider Dupré, D. 2018. Defeasible Reasoning in SROEL from Rational Entailment to Rational Closure. Fundam. Inform. 161, 1-2, 135-161.

Giordano, L. AND Theseider Dupré, D. 2020. An ASP approach for reasoning in a conceptaware multipreferential lightweight DL. CoRR abs/2006.0438\%.

GLIozzI, V. 2016. Reasoning about multiple aspects in rational closure for DLs. In Proc. AI*IA 2016, Genova, Italy, Nov 29 - Dec 1, 2016. LNCS, vol. 10037. 392-405.

Gottlob, G., Hernich, A., Kupke, C., And Lukasiewicz, T. 2014. Stable model semantics for guarded existential rules and description logics. In Proc. 14th Int. Conf. on Principles of Knowledge Representation and Reasoning, KR 2014, Vienna, Austria, July 20-24.

Kazakov, Y., Krötzsch, M., And Simancik, F. 2014. The incredible ELK. J. Autom. Reasoning 53, 1, 1-61.

Knorr, M., Hitzler, P., And Maier, F. 2012. Reconciling OWL and non-monotonic rules for the semantic web. In ECAI 2012. 474-479.

Kraus, S., Lehmann, D., and Magidor, M. 1990. Nonmonotonic reasoning, preferential models and cumulative logics. Artificial Intelligence 44, 1-2, 167-207.

Krötzsch, M. 2010. Efficient inferencing for OWL EL. In Proc. JELIA 2010. 234-246.

Lehmann, D. And Magidor, M. 1992. What does a conditional knowledge base entail? Artificial Intelligence 55, 1, 1-60.

Lehmann, D. J. 1995. Another perspective on default reasoning. Ann. Math. Artif. Intell. 15, 1, 61-82.

Lieto, A. And Pozzato, G. 2018. A description logic of typicality for conceptual combination. In Proc. ISMIS 2018, Cyprus, October 29-31, 2018. LNCS, vol. 11177. Springer, 189-199.

Motik, B. And Rosati, R. 2010. Reconciling Description Logics and rules. J. ACM 57, 5.

Pearl, J. 1990. System Z: A Natural Ordering of Defaults with Tractable Applications to Nonmonotonic Reasoning. In TARK (3rd Conference on Theoretical Aspects of Reasoning about Knowledge), R. Parikh, Ed. Morgan Kaufmann, Pacific Grove, CA, USA, 121-135.

Pensel, M. And Turhan, A. 2018. Reasoning in the defeasible description logic $E L_{\perp}$ - computing standard inferences under rational and relevant semantics. J. Approx. Reasoning 103, 28-70.

Straccia, U. 1993. Default inheritance reasoning in hybrid KL-ONE-Style Logics. In Proc. 13th Int. Joint Conf. on Artificial Intelligence (IJCAI 1993). Chambéry, France, 676-681. 